\title{
Comparative study on connection performance of steel joints in the prefabricated steel reinforced concrete shear wall
}

\author{
GAO Lin ${ }^{1,2}$, XIAO Xian ${ }^{1}$, GUO Kaixuan ${ }^{1}$, LIU Kunxiong ${ }^{1}$ \\ ${ }^{1}$ College of Civil and Architectural Engineering, North China University of Science and Technology, Tangshan 063210, China \\ ${ }^{2}$ Earthquake Engineering Research Center of Hebei Province1, Tangshan 063210, China
}

\begin{abstract}
In order to respond to the call of the strategy to vigorously carry out the fabricated structure, a new lateral connection joint- prefabricated steel joint with grouting bolt sleeve, which is adopted in the prefabricated steel reinforced concrete shear wall structure. Through uniaxial tensile test and finite element analysis, the prefabricated steel joint is comprehensive analysed, to get the impact trend of the anchorage length, sleeve wall thickness, bolt diameter to the connection performance, to determine the practicability and reliability of the connection way, and to ensure connection performance in the prefabricated steel reinforced concrete shear wall structure system. With the comparative studies, we find that, the finite element analysis and test results have high fitting degree, and can be verificated each other; the bolt diameter, sleeve wall thickness and anchor length all have positive effect trend on the tensile properties of the joint, but the influence decreases; The bolt diameter should be more than $12 \mathrm{~mm}$, the sleeve wall thickness should be more than $5 \mathrm{~mm}$, and the anchorage length should be more than $70 \mathrm{~mm}$; the shear bond diameter can reduce the diameter, and can change the section setting to the ring setting; the comparative results can provide reliable data support for the prefabricated steel connection joint design.
\end{abstract}

\section{Preface}

In the process of rapid urban development, the shortage of land resources, high construction costs, and lagging implementation of engineering technology have restricted the development of construction industrialization. In response to the national strategy for building energy conservation and emission reduction, many researchers have taken prefabricated building research as a breakthrough direction to break the bottleneck of the development of building industrialization. They have carried out extensive and in-depth research in related structural systems, engineering technology, and promotion and application, and have achieved fruitful results. The research results ${ }^{[1-2]}$.In the prefabricated steel concrete shear wall structure system, the horizontal connection between the upper and lower prefabricated shear wall pieces adopts a new connection method-grouting pin and sleeve type assembled steel connection node. This new type of steel node connection method uses a square steel sleeve to hoop the prefabricated shear wall at the horizontal end of the prefabricated shear wall. At the same time, a pin is used to cross the steel sleeve and the steel hoop area to form a bolt connection Effect, then through the grouting hole reserved on the steel sleeve, pour high-strength fiber grouting into the steel sleeve to form a grouting connection effect between the steel sleeve and the section steel hoop space, and finally realize the bolt connection and the grouting connection at the same time The effective combination and sufficient reinforcement of the fusion, to achieve the expected connection effect ${ }^{[3-4]}$.

Through the analysis of existing related research results, it can be found that the reliability of node connection is the key to realizing the prefabrication of building structures. The node connection technology with reasonable stress is the key technology of prefabricated structure. The practicality of node connection form, Convenience is also an important factor in determining whether it can be promoted and applied ${ }^{[5-6]}$. In this paper, we will analyze the influence of anchorage length, sleeve wall thickness, pin diameter and other factors on the mechanical performance of the joint connection form through the unidirectional tensile test and finite element analysis of the grouting pin sleeve assembly steel connection node. Trend, so as to more intuitively clarify the stress mechanism of this type of joint connection and the main influencing factors of the bearing capacity performance, so as to provide a reasonable structural measure for the fabricated steel connection node and the design method of the prefabricated steel concrete shear wall Good test data and application analysis reference.

\section{Research overview}

\subsection{Test parameters}

*Corresponding author's e-mail: 563473334@qq.com 
The test adopts the joint uniaxial tension method; the sleeve adopts Q235-grade square steel pipe with an outer diameter of $120 \mathrm{~mm} * 120 \mathrm{~mm}$, the yield strength of the pipe wall steel is $235 \mathrm{MPa}$, the ultimate strength is $440 \mathrm{MPa}$, and the modulus of elasticity is $2.06 \times 105 \mathrm{MPa}$; the section steel adopts Q235 grade $100 \mathrm{~mm} * 100$ $\mathrm{mm} * 4.3 \mathrm{~mm} * 6.5 \mathrm{~mm} \mathrm{H}$-shaped steel, the tensile strength is only $235 \mathrm{MPa}$; the pin uses HRB400 grade steel, the tensile strength of $10 \mathrm{~mm}$ diameter is $470 \mathrm{MPa}$, the tensile strength of $12 \mathrm{~mm}$ diameter is $485 \mathrm{MPa}$, and the tensile strength of diameter $14 \mathrm{~mm}$ is $14 \mathrm{~mm}$. $495 \mathrm{MPa}$; The shear key uses HRB400-grade steel bars with a diameter of $10 \mathrm{~mm}$, and the tensile strength is $470 \mathrm{MPa}$;

The tensile strength of $12 \mathrm{~mm}$ in diameter is $485 \mathrm{MPa}$, and the tensile strength of $14 \mathrm{~mm}$ in diameter is $495 \mathrm{MPa}$; the shear key uses HRB400 steel bars with a diameter of $10 \mathrm{~mm}$, and the tensile strength is $470 \mathrm{MPa}$; the grouting material is made of special sleeve grouting material produced by Tangshan Polar Bear Building Materials. It is $80 \mathrm{MPa}$. The variable parameters of each test piece are shown in Table 1.

Table 1. The design parameters of Specimen

\begin{tabular}{lccc}
\hline node & $\begin{array}{l}\text { Anchor } \\
\text { length } \\
\text { /mm }\end{array}$ & $\begin{array}{l}\text { Sleeve } \\
\text { thickness } \\
\text { /mm }\end{array}$ & $\begin{array}{l}\text { Pin } \\
\text { diameter } \\
/ \mathrm{mm}\end{array}$ \\
\hline Anchor 70(ordinary) & 70 & 5 & 12 \\
Anchor 70(thick) & 70 & 8 & 12 \\
Anchor 80(ordinary) & 80 & 5 & 14 \\
Anchor 80(thick) & 80 & 8 & 14 \\
Anchor 90(ordinary) & 90 & 5 & 12 \\
Anchor 90(thick) & 90 & 8 & 12 \\
Anchor 100 (ordinary) & 100 & 3 & 10 \\
Anchor 100 (thick) & 100 & 6 & 10 \\
\hline
\end{tabular}

\subsection{Finite element analysis parameters}

The finite element analysis adopts ABAQUS (6.13-1) software. When modeling, the gap between the pin and the pin hole is ignored, and the threaded pin is simplified into a cylinder; the set tensile strength of the sleeve and section steel are both $235 \mathrm{MPa}$, and the pin is Set to three-level steel bar (HRB400), the steel density is $7.8 \times 10^{-9} \mathrm{t} / \mathrm{mm}^{3}$, the Poisson's ratio is 0.3 , the Mises yield criterion is selected for the steel, the isotropic strengthening model is selected for monotonic load, and the steel constitutive model is elastic- Enhanced double broken line model; set compressive strength of grouting material is $80 \mathrm{MPa}$, density is $2.4 \times 10^{-9} \mathrm{t} / \mathrm{mm}^{3}$, elastic modulus is $2.95 \times 104 \mathrm{MPa}$, Poisson's ratio is $0.3^{[7]}$, and the constitutive model is Han Linhai.

\section{Comparative analysis of results}

\subsection{Destruction form}

During the test, the H-shaped steel was broken by comparing the results of observation test and finite element analysis. The finite element simulation results showed that the stress concentration around the pin bore It is more obvious than the test pieces with other anchoring lengths, and the stress concentration area is highly consistent with the shape of the test. The end of the pin is bent, and the grouting body is partially separated from the sleeve. The results reflected by the finite element simulation are highly similar; The pin penetrates through the sleeve walls on both sides and slightly bulges, the end of the pin is bent, and the grout part is separated from the sleeve. The phenomenon of finite element simulation is also highly consistent. It can be shown that the failure modes of the test and the finite element analysis are in good agreement overall, and the finite element software can more intuitively reflect the possible failure modes of the nodes, which can better guide the experimental research process and predict the possible results.

\subsection{Analysis of tensile bearing capacity}

Among the comparison curves of the tensile bearing capacity of the test and finite element analysis of each specimen, the abscissa represents the sliding displacement of the section steel and the sleeve, and the ordinate represents the tensile bearing capacity; in the test, after analysis of the later results, it was found that the measurement value was caused by the production error in the specimen production process and the measurement error in the measurement process On the high side, based on the principle of respecting reality, the measured value has been modified as necessary to obtain the tensile strength value used in the article.

The comparative analysis of tensile bearing capacity of test and finite element analysis found that:The test curves are slightly higher than the finite element analysis curve. This is because the pins selected for the test are all ribbed steel bars, and the finite element model uses a solid cylinder (without ribs), which results in the test pin diameter slightly larger than the finite element simulation In the finite element analysis, the contact between the grout and the sleeve wall is slightly lower than the bond strength in the test.Compared with the test curve, the finite element analysis curve has a faster growth in the early stage and reached the peak of the tensile bearing capacity earlier. This is due to the gap error in the production of the specimen in the test and the vacant deformation during the load process, while the finite element analysis There is no gap between the components, which eliminates the influence of vacant deformation, which leads to a faster increase in the tensile capacity of the finite element analysis, and the tensile capacity reaches its peak earlier.Compared with the test curve, the finite element analysis curve is more stable after reaching the ultimate tensile bearing capacity, and the decline is slow. This is because the double-broken line constitutive model of the steel is selected in the finite element analysis, which is different from the actual load-displacement curve of the steel. And no fracture model was input in the finite element analysis, which caused a slow decline in the later stage of 
the tensile capacity curve. The error of the ultimate tensile bearing capacity in the test and finite element analysis curves is within $20 \%$, most of which are around $10 \%$; the error of the anchoring length of $100 \mathrm{~mm}$ is the smallest of all anchoring lengths, only $8.3 \%-8.7 \%$, two The fit of the curve is the closest, and the finite element analysis has the best fitting effect.

\subsection{Nodal deformation analysis}

The comparative analysis of joint deformation of test and finite element analysis found that:The grouting body has the phenomenon of being pulled out of the sleeve, and the displacement of the ordinary sleeve is more obvious than that of the thickened sleeve, which is clearly reflected in the test and finite element analysis; the sleeve wall has a slight swelling phenomenon, and the ordinary sleeve The degree of bulging of the sleeve is more obvious than that of a thickened sleeve. The finite element analysis shows that the stress distribution on the sleeve wall shows that the stress concentration of ordinary sleeves is more serious than that of thickened sleeves. This shows that the thickness of the sleeve determines the sleeve. The ability of the barrel to restrain the grouting body also determines the ability of the sleeve to resist deformation under load.In the finite element analysis, the stress concentration phenomenon near the pin hole is obvious. The stress concentration phenomenon of the ordinary sleeve is more serious than that of the thickened sleeve. The corresponding test phenomenon is that the pin hole of the ordinary sleeve appears to different degrees Tear; as the diameter of the pin increases or as the anchoring length decreases, the stress concentration near the pin hole becomes more serious (e.g. the stress concentration phenomenon of the diameter of $14 \mathrm{~mm}$ is more common than that of the diameter of $12 \mathrm{~mm}$. Serious; if evaluated according to the anchoring length, the severity of stress concentration is $70 \mathrm{~mm}, 80 \mathrm{~mm}$, and $90 \mathrm{~mm}$ in order of anchor length. The test also reflects that the hole and tear of the corresponding test piece is more serious; this indicates The wall thickness of the sleeve determines the pressure-bearing capacity of the sleeve against the pin, and the diameter of the pin determines the shear resistance of the joint, and further determines the tensile bearing capacity of the joint.

In the finite element analysis, it can be seen that the bending degree of the pin of the ordinary sleeve is more obvious than that of the thickened sleeve, and the degree of bending decreases with the increase of the diameter of the pin, which corresponds to the experimental phenomenon; this is due to In the case of ordinary sleeves, the proportion of the load shared by the pin is higher, and the bending of the pin is more obvious, but as the diameter of the pin increases, the shear resistance of the pin after being loaded increases, and the degree of bending of the pin is greater. Light; this shows that the diameter of the pin determines the shear resistance and the joint tensile capacity.

\section{4 conclusion}

According to the comparative study of the test results and the finite element analysis, it can be seen that the connection performance of the fabricated steel nodes can meet the requirements. The finite element analysis can make up for the detailed analysis of the experimental research stage and form a mutual reflection; and draw a conclusion about the fabricated steel The feasibility conclusion of connecting nodes.

(1) The degree of influence on the influencing factors of node connection performance are: pin diameter, sleeve wall thickness, and degree of anchorage.

(2) The diameter of the pin has a positive effect on the tensile bearing capacity of the node, and it also determines the degree of damage to the node, especially the stress condition of the pin hole. It is recommended to set the pin diameter to $12 \mathrm{~mm}$ or more.

(3) The wall thickness of the sleeve has a positive effect on the tensile bearing capacity of the joint, and it also determines its ability to restrain the grouting body, especially the deformation of the sleeve wall. It is recommended that the sleeve wall thickness should be more than $5 \mathrm{~mm}$. Or the sleeve wall thickness strengthening measures can be taken on the side of the pin hole to strengthen the coordinated shear performance of the pin and bolt, thereby improving the tensile bearing capacity of the node.

(4) The anchorage length has a positive effect on the tensile bearing capacity of the node, which determines the bonding ability with the grouting body, especially the reasonable distance between the pin and the drill hole. It is recommended that the anchorage length should be $70 \mathrm{~mm}$ or more. should.

(5) The welded shear key on the inner wall of the sleeve and the end of the section steel has a positive effect on the tensile bearing capacity of the node, which determines the coordinated bearing effect between the sleeve, section steel and grouting body. It is recommended to set the segmented shear key to The ring-shaped shear key can reduce the diameter of the shear key while ensuring the connection performance of the nodes.

\section{References}

1. Jiang Qinjian. Research report on the development of China's construction industry. Concrete World, 2014; 7: 10-20

2. Yan Wei, Cao Yonghong, Li Guorong. Development of prefabricated structural system and building industrialization. Journal of Chongqing Jianzhu University, 2004; 10(5): 131-133

3. Gao Lin, Chen Jianwei, Chen Haibin, etc. Connection structure of prefabricated steel-concrete components: 201620154101.9. 2016-7-6

4. Gao Lin, Chen Jianwei, Chen Haibin, etc. Prefabricated steel-concrete component connection structure and its construction method: 201610114037.6. 2017-7-7 
5. Chen Jianwei, Su Youpo, Chen Haibin, et al. Research progress on key technologies of prefabricated shear wall with frame. Journal of Hebei Union University (Natural Science Edition), 2013; 35(3): 96-101

6. Chen Jianwei, Su Youpo. Prefabricated shear wall structure and its connection technology. World Earthquake Engineering, 2013; 29(1): 38-48

7. Wu Wenping, Huang Bingsheng, Fan Jianhui. Research on 3 different constitutive relationship models of concrete filled steel tube[J]. Sichuan Building Science Research, 2009; 35(6): 19-23

8. Han Linhai. Concrete-filled steel tube structure [M]. Beijing: Science Press, 2000 